Artículo de investigación.

Cómo citar: Montes, M., y Rodelo, F. (2019). Absueltos y oprimidos: representaciones mediáticas de las personas con discapacidad en México. MEDIACIONES, 15(22),

23-42. https://doi.org/10.26620/uniminuto. mediaciones.15.22.2019.23-42

Editorial: Corporación Universitaria Minuto de Dios - UNIMINUTO.

ISSN: $1692-5688$

eISSN: 2590-8057

Recibido: 12 de julio de 2018

Aceptado: 19 de septiembre de 2018

Publicado: 15 de marzo de 2019

Conflicto de intereses: los autores han declarado que no existen intereses en competencia.

\section{Absueltos y oprimidos: representación mediática de las personas con discapacidad en México ${ }^{1}$}

\author{
Absolved and oppressed: media representation \\ of people with disabilities in Mexico
}

\section{Absolvido e oprimido: representação midiática das pessoas com deficiência no México}

\section{Resumen}

Los periódicos representan a las personas con discapacidad apegándose a dos modelos principales: el médico, que percibe la discapacidad como una enfermedad que incapacita para actividades cotidianas, o el social, que entiende la discapacidad como una imposición de obstáculos sociales sobre una persona que tiene una limitación física, mental o sensorial. Para averiguar cómo representan los medios en México a este grupo y sus problemas, se realizó un análisis de contenido de los artículos sobre discapacidad publicados durante 2015 en cuatro periódicos. Se encontró que estos tienden a presentar una perspectiva paternalista sobre las personas con discapacidad, representándolas como sujetos desempoderados y cuya socialización no propicia inclusión social auténtica.

\section{Palabras clave}

Discapacidad, modelo social, medios de comunicación, inclusión, discriminación.
Frida Viridiana Rodelo Amezcua frida.rodelo@academico.udg.mx Universidad de Guadalajara México martha.garimontes@gmail.com

Universidad de Guadalajara

México
1 Este artículo se deriva de la investigación homónima para obtener el grado de maestra en Comunicación por la Universidad de Guadalajara, realizada por Martha Garín y dirigida por la doctora Frida V. Rodelo. Fue desarrollada entre septiembre de 2014 y diciembre de 2016. Este trabajo fue financiado por el Consejo Nacional de Ciencia y Tecnología (Conacyt). 


\section{Abstract}

Newspapers represent people with disabilities by adhering to two main models: the medical model, which perceives disability as an illness that incapacitates a person from fulfilling daily activities, or the social model, which understands disability as an imposition of social obstacles on a person who has a physical, mental or sensory impairment. In order to understand how the media in Mexico represents this group and its problems, a content analysis of articles on disability published during 2015 in four newspapers was carried out. It was found that these tend to present a paternalistic perspective on people with disabilities, representing them as disempowered subjects whose socialization does not propitiate authentic social inclusion.

Palabras clave

Disability, social model, media, inclusion, discrimination.

\section{Resumo}

A forma como os jornais representam as pessoas com deficiência normalmente segue dois modelos principais: o modelo médico, que percebe a deficiência como uma doença que incapacita para as atividades diárias, ou o modelo social, que entende a deficiência como uma imposição de obstáculos sociais a uma pessoa com deficiência física, mental ou sensorial. A fim de descobrir como a mídia mexicana representa este grupo e seus problemas, realizou-se uma análise do conteúdo dos artigos sobre deficiência publicados durante 2015 em quatro diferentes jornais. Constatou-se que estes tendem a apresentar uma perspectiva paternalista sobre as pessoas com deficiências e incapacidades, representando-as como sujeitos desempoderados cuja socialização não propicia uma autêntica inclusão social.

\section{Palavras-chave}

Deficiência, modelo social, mídia, inclusão, discriminação. 


\section{Introducción}

Ante las pocas oportunidades de convivencia pública de las personas con discapacidad (en adelante PCD), los contenidos de los medios de comunicación son una manera de conocer a las PCD, sus necesidades y problemas, así como una vía para saber cómo darles un trato adecuado y justo. Por esto es posible decir que las organizaciones de medios funcionan como educadores sociales en el tema de la discapacidad al publicar de manera masiva artículos informativos que abordan esta condición y ofrecer posturas ideológicas que permiten a los miembros de la sociedad generarse opiniones sobre la discapacidad (Haller \& Burns, 2015). Además de esto los medios actúan como generadores o limitadores de oportunidad, al influenciar la manera en que las PCD piensan sobre sí mismas (Zhang \& Haller, 2013). Las perspectivas teóricas de la construcción social (Berger \& Luckmann, 1968) y el estigma social (Goffman, 2008) consideran también que la formación y el sostenimiento de las identidades se basan en su aceptación y asimilación por parte de los miembros de una sociedad, proceso en que los medios contribuyen de forma poderosa.

La mayoría de los estudios a nivel internacional que analizan la cobertura mediática de la discapacidad se centran en el análisis de las coberturas de las paralimpiadas, así como en casos de movilización por los derechos de las PCD. Sin embargo, el estudio de la discapacidad desde el campo de la ciento de su población tiene alguna discapacidad (Inegi, 2013). Para conocer cómo es la representación de la condición de discapacidad en los periódicos mexicanos, se realizó un análisis de contenido de los cuatro periódicos de prestigio más importantes de la ciudad de Guadalajara. De acuerdo con los resultados obtenidos, las PCD son representadas como no responsables de sus problemas, personas no incluidas ni criticadas, segregadas y desempoderadas. En otras palabras, como individuos absueltos y oprimidos. Este artículo plantea una crítica frente a las representaciones mediáticas de la discapacidad que más que fomentar un sentimiento de solidaridad y empatía hacia las PCD proyectan una imagen en la que aparecen como sujetos necesitados de la ayuda e incapaces de hacer algo para resolver sus propios problemas; de esta manera contribuyen a mantener una imagen social de las PCD que las posiciona como sujetos impedidos.

\section{Marco teórico}

En su obra Estigma, Goffman (2008) aborda diferentes situaciones, entre estas la discapacidad, que implican la portación de un estigma social, el cual define como “...un atributo profundamente desacreditador" (2008, p.15) o "una clase especial de relación entre atributo y estereotipo” (2008, p. 16). De esta forma identifica dos clases de sujetos en un espacio social: los estigmatizados, y los 
normales, siendo estos últimos "todos aquellos que no se apartan negativamente de las expectativas particulares que están en discusión” (Goffman, 2008, p. 17). Goffman señala que el rasgo que estigmatiza a la persona puede atraer tan fuertemente nuestra atención que el resto de sus atributos son anulados, lo cual puede provocar un alejamiento de esa persona; además señala que es posible llegar a considerar que la persona estigmatizada no es completamente humana (2008, p. 17), lo cual, a su vez, deriva en prácticas de discriminación. Tal “atracción” que el "estigma” de las PCD ejerce sobre los otros está ligada a la familiaridad con ese tipo de rasgos, lo cual se relaciona con la presencia pública de estas personas o con las representaciones de ellas.

Goffman (2008) señala que las personas que tienen un estigma físico refieren que las personas consideradas como normales con las que tienen contacto continuo lograrán aceptar su condición, creando una especie de "rutina diaria de normalización” (p. 73). Añade que "es probable que cuanto mayor sea la alianza del individuo con los normales, más se considerará a sí mismo en términos no estigmáticos” (p. 137).

De acuerdo con Goffman, las afiliaciones de la persona estigmatizada a ciertos grupos tienen efectos en su interacción y en la forma en que son percibidos por los demás. Esta concepción teórica tiene paralelo en Berger y Luckmann (1968), quienes sostienen que "el individuo llega a ser lo que los otros significantes lo consideran” (Berger \& Luckmann, 1968, p. 165). Berger y Luckmann entienden por socialización "la inducción amplia y coherente de un individuo en el mundo objetivo de una sociedad o en un sector de él" (1968,p. 164). En el caso de las PCD puede ocurrir lo que los autores denominan socialización deficiente, la cual "ocurre solo como resultado de accidentes biográficos, ya sean biológicos o sociales” (p. 204) y que implica una asimetría entre "la propia realidad subjetiva" de la persona y "la realidad socialmente definida”, y que da lugar a "antidefiniciones”, que aparecen en el momento en que los individuos se organizan en grupos (1968, p. 205). Berger y Luckmann indican que el sujeto construye significados de sí mismo a partir de lo que los demás creen que es. Por esto, los grupos organizados de personas que crean “antidefiniciones” ofrecen una alternativa para tener una socialización exitosa dentro del grupo, ya que mientras no lo hagan serán solamente portadores de la definición que los otros les adjudican (1968, p. 206).

Berger y Luckmann señalan que "los otros" funcionan como "coro", que confirma la realidad en la que nos desenvolvemos (1968, pp. 186-188) pues la opinión de alguien próximo a una persona tendrá peso en ella e influirá en la construcción de su identidad. A la vez advierten que existe la posibilidad de que ocurra una disconfirmación de la realidad subjetiva que ejemplifican de la siguiente manera: "una misma opinión expresada sucesivamente por diez conocidos míos casuales puede llegar a contrarrestar la opinión contraria de 
mi mejor amigo" (1968, p. 188). Los medios de comunicación pueden constituir otra forma de confirmar o disconfirmar la realidad, al ser una especie de "coro", o un elemento que difunde una perspectiva y que es respaldada por un "coro", que en este caso es la audiencia que consume los contenidos de los medios.

Las perspectivas teóricas de Berger y Luckmann (1968) y Goffman (2008) se orientan a explicar la construcción de la realidad social y la identidad a través de la interacción social; también, permiten ponderar cómo las representaciones mediáticas de los sujetos, que reflejan concepciones sociales imperantes respecto de la discapacidad, contribuyen a partir de una continua dialéctica a posicionar y mantener cierta imagen social de las PCD.

\section{Representaciones mediáticas de la discapacidad}

Existen distintos modelos para pensar y actuar ante el tema de la discapacidad. En los contenidos de los medios de comunicación, von Krogh (2010) distingue las perspectivas médicas, social o de derechos humanos ${ }^{2}$, mientras que Cuesta et al. (2015) identifican representaciones de discapacidad basadas en los modelos tradicional, rehabilitador (que comparte muchas de sus características con el médico) y social. Los estudios que se proponen el señalar qué perspectiva es más común en la cobertura periodística de temas relacionados con la discapacidad y las PCD son escasos. De entre estos, Cuesta et al. (2015) encontraron que es menor la cantidad de artículos periodísticos en la prensa española los que presentan encuadres relativos al modelo social de la discapacidad en comparación con los modelos tradicional y rehabilitador. Entre las notas de modelo tradicional, los autores encontraron en mayor medida las que exaltan "la discapacidad como tragedia personal" (Cuesta et al., 2015, p. 276), estigmatizando así la condición de discapacidad al dar sustento a la idea de apartar a las personas con esta condición del resto de la sociedad, lo cual resulta completamente contrario a lo planteado por los modelos social y de Derechos Humanos.

Además de la identificación del modelo prevaleciente en la representación mediática de la discapacidad, otros enfoques seguidos en la investigación sobre el tema son el estudio de las etiquetas para nombrar la discapacidad en los medios (en inglés, media labeling) y el estudio de encuadres en la cobertura de la discapacidad.

2 De acuerdo con este mismo autor, la perspectiva médica ubicaría la causa del problema en la PCD, a quien se le ve como un paciente. La perspectiva social encontraría la causa del problema en la sociedad. Una perspectiva de derechos humanos, por su parte, enfatizaría la necesidad de lograr los mismos derechos para todos (Von Krogh, 2010). 


\section{Uso de etiquetas para nombrar la discapacidad.}

En el contexto posterior a la firma del Americans with Disabilities Act, Haller et al. (2006) examinaron el uso de etiquetas para referir la discapacidad en los medios de comunicación. De acuerdo con los autores, uno de los puntos promovidos por el Disability Rights Movement había sido el uso de un lenguaje apropiado para referir la discapacidad (Haller et al., 2006). El lenguaje es un campo donde pueden darse confrontaciones, cuyos resultados son maximizados por los medios de comunicación. Un ejemplo de lo anterior es la tensión referida por Haller et al. (2006) entre el "orgullo" de las PCD y la narrativa preferida del resto de las personas, donde se considera la discapacidad como una adversidad y la PCD como una persona "especial" por tener que afrontarla, refiriendo de la manera siguiente la preferencia de las organizaciones de medios por las narrativas de interés humano: "El movimiento por los derechos de la discapacidad está tratando de avanzar una agenda política seria, mientras que los medios de comunicación se centran en las personas ciegas que juegan a los bolos o en un adolescente con desfiguración facial grave que enfrenta la vida con gracia” (p. 63). Centrándose en las etiquetas propuestas por los medios, Haller et al. (2006) discuten:

- Las connotaciones negativas de términos como lisiado (cripple) y discapacitado (disabled).

- La posibilidad de resignificación de los términos, evidenciada en el uso de las palabras raro (queer) y negro (black) y su asociación con la dignidad y la agencia de los grupos referidos.

- La identificación de términos, clichés y frases hechas para referir la discapacidad que otorgan mayor importancia a la condición que a la persona: "atado a una silla de ruedas", "víctima de sida", "paciente de sida”. Y, en contraposición, la posibilidad de utilizar términos que ponen "primero" a la persona (people-first terms): "persona con discapacidad", "persona con sida".

En el contexto hispanohablante, Iáñez y González (2017) encontraron en los periódicos de prestigio españoles la persistencia de etiquetas que hoy en día se consideran peyorativas, como minusválido, y como principal tendencia actual los términos derivados de discapacidad (en primer lugar, discapacitado; pero también persona discapacitada y persona con discapacidad).

\section{Encuadres en las notas sobre discapacidad.}

Otras investigaciones se han propuesto abarcar objetos adicionales a las etiquetas de la discapacidad, con el interés primordial de describir el 
tratamiento periodístico de desórdenes mentales como el autismo debido al estigma social del que son objeto. McKeever (2013), por ejemplo, midió los encuadres sobre discapacidad, las fuentes periodísticas, las causas sugeridas y las soluciones propuestas en artículos de periódicos de prestigio estadounidenses publicados en el periodo comprendido entre 1996 y 2006. De esta manera, concluyó que en el tratamiento del autismo en los periódicos de élite norteamericanos analizados predominaron las notas con encuadre "humano" (human frame). De acuerdo con la autora, el optar por este tipo de tratamiento no tendría la consecuencia negativa de exonerar a los actores sociales (gobierno, sociedad) por este problema, como sí ha sido establecido para el caso de la cobertura de la pobreza (Iyengar, 1990). Más bien, “estas historias de interés humano pueden ayudar a llamar la atención acerca de enfermedades o desórdenes incluso entre los creadores de políticas públicas, y esto es especialmente verdadero con el autismo” (p. 231). Las fuentes más utilizadas en los artículos analizados por McKeever procedieron del gobierno y del sector médico, mientras que un porcentaje relativamente bajo de artículos presentaban como fuentes a las propias personas con autismo (McKeever, 2013, p. 229).

Varios investigadores se han centrado en estudiar las características de la cobertura noticiosa de las paralimpiadas (tal vez), por ofrecer este evento internacional una ocasión única para observar la representación de la discapacidad a la vez de permitir establecer una comparación frente a la cobertura de las olimpiadas. Resultados recurrentes en diferentes análisis de las coberturas de estos eventos deportivos son la construcción de los participantes en "otros” (en inglés, othering) a través del énfasis en la excepcionalidad del atleta con discapacidad o el superhumano (superhuman o supercrip, que supera obstáculos descomunales) (Silva \& Howe, 2012; Misener, 2013); el tono exageradamente positivo de los artículos (Howe, 2008; Carter \& Williams, 2012); la desmesurada preferencia por artículos de interés humano (Ellis, 2008; Carter \& Williams, 2012) y la evasión de temas políticos (Carter \& Williams, 2012).

Otro tipo de asuntos estudiados en los análisis de cobertura mediática son las movilizaciones, protestas e iniciativas a favor de los derechos de las PCD. Por ejemplo, a partir del análisis de la cobertura mediática de protestas de PCD en Israel, Rimmerman y Herr (2004) describen y evalúan la fortaleza del movimiento en ese país. Haller y Burns (2015) analizaron la cobertura de la aprobación del Esquema Nacional de Seguro de Discapacidad en Australia y descubrieron que, pese a que la meta de la ley era contribuir a que las PCD construyeran vidas independientes, los contenidos mediáticos comunicaban el asunto reproduciendo modelos tradicionales de discapacidad y proyectado una imagen de las PCD como personas en desventaja necesitadas de ayuda económica de terceros (p. 272). 
También desde la perspectiva de encuadres, Maia y Vimieiro (2015) buscaron describir el "progreso moral” en la representación mediática de la discapacidad en Brasil, en un estudio longitudinal que toma como referencia teórica los planteamientos de Honneth con respecto al progreso moral y el reconocimiento. Maia y Vimieiro (2015) analizaron las tensiones y conflictos que emergen del proceso de inclusión de las PCD en diferentes ámbitos de la vida social y cómo se reflejan en las noticias que analizan, obteniendo como resultado que el trascurrir de los años ha traído un progreso moral con respecto al tratamiento del tema de la discapacidad manifestado en una mayor preocupación por el tema de la inclusión y un mayor entendimiento de la necesidad de incluir a las PCD y su expresión de necesidades en los medios de comunicación, que sin embargo no han logrado eliminar las tensiones y conflictos que rodean la vida social de las PCD.

\section{México: modelo de discapacidad y medios de comunicación.}

En México habitan 5’739.270 personas (5,1 por ciento de la población) con diversos tipos de discapacidad (Inegi, 2013), de las cuales apenas una pequeña parte cuenta con las oportunidades suficientes para desarrollar cualidades y capacidades personales que les ayuden a vivir plenamente: las cifras del Inegi (2013) dan cuenta de que las PCD en México constituyen una minoría con acceso limitado a educación y empleo, por lo que puede afirmarse que la sociedad y el Estado mexicanos no se han preparado para incluir eficazmente a las PCD en la sociedad a la cual pertenecen y en la cual tienen derecho a participar activamente produciendo beneficios para esta misma y disfrutando de lo que la convivencia social les ofrece, con todo y que la Ley General para la Inclusión de las Personas con Discapacidad establece en su artículo 4 que la PCD es acreedora de todos los derechos inherentes al ciudadano mexicano y que:

las medidas contra la discriminación consisten en la prohibición de conductas que tengan como objetivo o consecuencia atentar contra la dignidad de una persona, crear un entorno intimidatorio, hostil, degradante u ofensivo, debido a la discapacidad que ésta posee.

El médico y el social son los principales modelos para concebir la discapacidad. Shakespeare (2006) indica que el modelo médico se basa en una ideología que tiende a reducir "...los complejos problemas de las personas con discapacidad a temas de prevención médica, cura o rehabilitación” (p. 199). El modelo social de la discapacidad, por su parte, nació como resultado de la lucha de diversos movimientos de organizaciones de PCD en Gran Bretaña y entiende la discapacidad como una imposición social sobre las personas con impedimentos físicos (Shakespeare, 2006). Dichos modelos son la base de actitudes y hábitos 
frente a la discapacidad y sirven para identificar cuál es la naturaleza de las acciones que toman los gobiernos, las instituciones y los individuos para atender a las PCD e interactuar con ellas; asimismo, nos permiten conocer si el trato que se les da propicia una actitud de asistencialismo o de reconocimiento como sujetos activos en la sociedad a la que pertenecen.

Aunque el contexto legislativo mexicano está anclado en la perspectiva del modelo social de la discapacidad a partir del compromiso del Estado mexicano con la Convención sobre los Derechos de las Personas con Discapacidad, esto no implica que la ideología imperante en la sociedad mexicana y sus organizaciones e instituciones se correspondan con dicha concepción. La Ley General para la Inclusión de las Personas con Discapacidad publicada en 2011 señala en su artículo 2 que persona con discapacidad (PCD) es:

Toda persona que por razón congénita o adquirida presenta una o más deficiencias de carácter físico, mental, intelectual o sensorial, ya sea permanente o temporal y que al interactuar con las barreras que le impone el entorno social, pueda impedir su inclusión plena y efectiva, en igualdad de condiciones con los demás.

Atendiendo a tal ley, debe entenderse la discapacidad como una condición de la persona que al entrar en contacto con el ambiente social puede provocar algunas dificultades en su inclusión social. Esta definición, por tanto, se adscribe al modelo social de la discapacidad (Shakespeare, 2006), que concibe la discapacidad como una imposición social y no una condición inherente a la persona con un impedimento (Shakespeare, 2006). No obstante, pese a este sustento jurídico, la convivencia y el desarrollo educativo, laboral y, por tanto, la dimensión social de la vida de las PCD se ve atrofiada debido a diversas manifestaciones de discriminación y desconocimiento de la discapacidad (Espinola-Nadurille \& Delgado, 2009). Ante esta situación, los medios de comunicación aparecen como agentes socializadores relevantes al divulgar masivamente representaciones de las PCD y los problemas que enfrentan a través de las noticias que reportan sobre ellas.

Asimismo, la falta de inclusión en el sistema educativo mexicano, al persistir en México la educación especial (Comité sobre los Derechos de las Personas con Discapacidad, 2014), incide en que sean los medios de comunicación los actores con mayor influencia en la socialización del tema de la discapacidad. Esto no es soslayado por la Convención sobre los Derechos de las Personas con Discapacidad, la cual en su artículo 8 impone a los Estados parte la obligación de sensibilizar a la sociedad con respecto a los derechos de las PCD, así como sus capacidades y aportaciones sociales, a través de intermediarios como lo son, entre otras instituciones sociales, los medios de comunicación. En concordancia, la Ley General para la Inclusión de las Personas con 
Discapacidad contempla el uso de los medios de comunicación para fomentar la independencia y desarrollo integral de las PCD (artículos 19-IV y 20), así como la libertad de expresión y de opinión de las PCD como forma de facilitar su participación e integración (artículo 32).

Frente a la escasez de estudios sobre la representación mediática de la discapacidad en los ámbitos mexicano y latinoamericano, nos propusimos realizarunainvestigación quepermita conocercómosonestasrepresentaciones. Los medios representan a las minorías mediante la problematización de un asunto público que se relaciona directamente con ellas, sin importar si es esta minoría la que simboliza un problema para la comunidad en la que vive o si es ella la que se ve aquejada por un problema que le afecta directamente.

Desde la perspectiva de encuadres de Entman (1993), encuadrar implica elegir ciertos elementos de la realidad para hacerlos más enfáticos dentro de un texto, con el objeto de definir un problema de un modo determinado, presentar una interpretación de sus causas, una evaluación o una solución para el mismo (Entman, 1993, p. 52). De esta manera, los encuadres en los asuntos de las PCD problematizados en los medios de comunicación implican maneras de definir sus problemas, la presencia de actores, de causas y soluciones para dichos problemas.

Los mensajes mediáticos pueden reforzar actitudes sociales, históricamente aceptadas y repetidas que impiden romper con el esquema establecido de la discapacidad para renovarlo con una perspectiva que conciba a las PCD como ciudadanos con derechos y obligaciones, como el resto de los miembros de la sociedad. En esta investigación se realizó un análisis de contenido de los periódicos de la ciudad de Guadalajara, México, con el objetivo de conocer y describir la representación mediática sobre la discapacidad y las personas con discapacidad en los periódicos mexicanos y determinar si es propiciadora de oportunidades, así como determinar de qué manera cumplen con la tarea de educar sobre este tema.

\section{Método}

Con el objetivo de conocer cómo son las representaciones mediáticas de la discapacidad y las PCD se realizó un análisis de contenido de los periódicos mexicanos. El análisis de contenido es un método sistemático y objetivo de análisis de la comunicación que busca identificar variables o referencias estables en el contenido de los textos (Wimmer \& Dominick, 2001, p. 135). La técnica del análisis de contenido ha sido frecuentemente utilizada para analizar la representación mediática de grupos minoritarios (Wimmer \& Dominick, 2001, p. 137). 
Selección de periódicos. Fueron analizados los artículos sobre discapacidad publicados durante 2015 en los cuatro periódicos generalistas más importantes de Guadalajara, segunda metrópolis mexicana más importante en términos de población: los tres periódicos de mayor tiraje (El Informador, El Occidentaly Mural) y uno de reciente circulación (Diario NTR Guadalajara). Cada uno de estos diarios tiene diferentes orientaciones ideológicas y perfil de lectores por lo cual se consideró que en conjunto permitirían indicar concepciones de discapacidad tanto en organizaciones como en lectores. Los tres periódicos de mayor circulación llegan a parte significativa de la población y cuentan no solo con una larga historia dentro del periodismo local, sino que son fácilmente identificados por los lectores, además de que simpatizan con las ideologías de grupos sociales dominantes. De entre estos, dos forman parte de empresas mediáticas de relevancia nacional (Grupo Reforma y Organización Editorial Mexicana), que sindican contenidos a múltiples publicaciones. Por otra parte, se incluyó a Diario NTR Guadalajara por ser un periódico que se autopresenta como crítico y que en corto tiempo ha ganado reconocimiento y prestigio.

Mural y El Occidental fueron analizados en sus versiones impresas a través de la base de datos Emerging Markets Information Service (Emis). El Informador y Diario NTR Guadalajara fueron analizados en sus versiones digitales. La muestra se compuso de todos los artículos periodísticos (noticia, breve, reportaje, artículo de opinión, entrevista) que mencionaron la palabra discapacidad y sus variantes en el periodo comprendido entre el 1 de enero y el 31 de diciembre de 2015, lo cual dio lugar a la recolección y posterior codificación de 583 notas.

\section{Libro de códigos y codificación}

Tomando como referencia a Rodelo y Muñiz (2016), el libro incluyó tres tipos de ítems: (a) Datos de identificación de la unidad de análisis: información que sirve para identificar la nota como una unidad que corresponde a un periódico, fecha o género periodístico específico. (b) Importancia de la nota: información que brinda una valoración de la importancia que tuvo la nota en el momento de su publicación, como lo es el espacio que ocupó, medido en número de palabras, o la sección en que se publicó. (c) Encuadres sobre la discapacidad: a partir de ítems dicotómicos (dos valores posibles: sí o no hay presencia), se recuperó información sobre los rasgos presentes en cada texto periodístico: tema de la nota, actores y tipos de discapacidad mencionados, cuáles son las causas del problema abordado en la nota, cuáles son las posibles soluciones y cuál es la evaluación moral que se hace de la discapacidad. La lista completa de variables y categorías se encuentra en la tabla 1. 
Tabla 1. Resultados por elemento de encuadre

\begin{tabular}{|c|c|}
\hline Variable & $\begin{array}{c}\text { Porcentaje } \\
\text { de notas con } \\
\text { rasgo }(n=583)\end{array}$ \\
\hline \multicolumn{2}{|c|}{ Definición del problema: temas relativos a discapacidad } \\
\hline Leyes, reglamentos o iniciativas & 41 \\
\hline Políticas públicas (programas de ayuda a PCD) & 30,5 \\
\hline Mejoras en la infraestructura & 19,2 \\
\hline Oportunidades educativas & 14,2 \\
\hline Oportunidades laborales & 9,8 \\
\hline Avances en dispositivos para mejorar condiciones físicas & 6,9 \\
\hline Oportunidades de escuelas especiales & 4,8 \\
\hline Avances en tratamientos de rehabilitación & 4,6 \\
\hline Donaciones en especie o actividades voluntarias & 4,3 \\
\hline Donaciones económicas a grupos de PCD & 3,1 \\
\hline Mejores en ingresos o condiciones laborales & 2,4 \\
\hline Condiciones económicas de pobreza de PCD & 2,1 \\
\hline Teletón & 1,9 \\
\hline Manifestaciones o protestas en favor de PCD & 0,5 \\
\hline \multicolumn{2}{|c|}{ Definición del problema: tipo de discapacidad mencionada } \\
\hline Discapacidad en general & 73,6 \\
\hline Visual & 23 \\
\hline Para moverse & 21,6 \\
\hline Intelectual & 10,6 \\
\hline Auditiva & 9,3 \\
\hline Para hablar & 2,2 \\
\hline \multicolumn{2}{|c|}{ Definición del problema: actores mencionados } \\
\hline Funcionamiento público o gobernante & 54,4 \\
\hline Otros actores (deportista, artistas, etc.) & 41,7 \\
\hline Activista, miembro de ONG, académicos o empresarios. & 25,7 \\
\hline Persona con discapacidad (PCD) & 24,4 \\
\hline Especialistas en leyes o de asociación civil & 16,5 \\
\hline Testimonio o experiencia de PCD & 14,8 \\
\hline Personas dedicadas a la lucha por las PCD & 9,3 \\
\hline Familiar o persona cercana a PCD & 8,6 \\
\hline Testimonio o experiencia de personas cercanas a PCD & 5,8 \\
\hline Médico especialista & 6 \\
\hline Actores políticos sin cargo oficial & 4,3 \\
\hline Terapeuta de discapacidad o rehabilitación & 1,9 \\
\hline
\end{tabular}

Continúa siguiente página... 
Continuación Tabla 1. Resultados por elemento de encuadre

\begin{tabular}{|l|r|}
\hline \multicolumn{1}{|c|}{ Variable } & \multicolumn{1}{|c|}{$\begin{array}{c}\text { Porcentaje } \\
\text { de notas con } \\
\text { rasgo (n=583) }\end{array}$} \\
\hline El gobierno es responsable & 22 \\
\hline La sociedad es responsable & 12,5 \\
\hline Las familias y las PCD son responsables & 4,6 \\
\hline Los empresarios o empleadores son responsables & 4,6 \\
\hline Las instituciones educativas son responsables & 4,1 \\
\hline Las instituciones médicas son responsables & 2,1 \\
\hline Las organizaciones de ayuda a las PCD son responsables & 0,7 \\
\hline \multicolumn{1}{|c|}{ Soluciones para resolver problemas relativos a la discapacidad } \\
\hline Leyes, reglamentos o políticas públicas & 46,8 \\
\hline Oportunidades educativas & 11,8 \\
\hline Avances médicos y tecnológicos & 10,8 \\
\hline Oportunidades laborales & 9,6 \\
\hline Acciones de las familias o personas cercanas a PCD & 8,4 \\
\hline Teletón & 1,5 \\
\hline Manifestaciones, protestas o huelgas & 0,3 \\
\hline \multicolumn{1}{|c|}{ Evaluación moral } & \\
\hline Expresa juicios positivos sobre la discapacidad & 3,4 \\
\hline Expresa juicios negativos sobre la discapacidad & 2,1 \\
\hline
\end{tabular}

Nota: Los elementos de encuadre no suman $100 \%$ porque se utilizaron variables dicotómicas.

Fuente: elaboración propia.

Se codificó la presencia de evaluaciones morales positivas a partir de la presencia de palabras o frases que enaltecen a las PCD o que las representan como extraordinarias (por ejemplo, "luchador"). Se identificaron como notas con evaluaciones morales negativas las que contienen palabras o frases que degradan a las PCD o que presentan la condición de discapacidad como limitante o impedimento para realizar actividades cotidianas, tales como estudiar o trabajar (por ejemplo, "no puede ir a la escuela porque tiene discapacidad").

El análisis de contenido de las notas fue realizado por tres codificadores. Para evaluar la fiabilidad intercodificador se midió el porcentaje de acuerdo (PA, también llamado fórmula Holsti) en cada variable a partir de la comparación de 
dos codificaciones realizadas por diferentes personas a una misma submuestra, la cual consistió en una muestra aleatoria simple de $10 \%$ de la muestra analizada en el estudio). Los PA obtenidos se ubicaron en un rango entre 56,8 \% (en la variable "otro tipo de actor") y 100 \% (en seis diferentes variables). La media aritmética del PA de las variables analizadas fue de $89.9 \%$, resultado que consideramos aceptable.

\section{Resultados}

Se analizaron 583 artículos publicados en 2015 en cuatro periódicos de Guadalajara y distribuidos a lo largo del año, con una cantidad mayor de notas publicadas en diciembre, mes en que se conmemora el día internacional de las PCD. El análisis de contenido de notas periodísticas (ver tabla 1) reveló que el tema con mayor incidencia en las notas analizadas fue el de leyes, reglamentos o iniciativas (41\% de las notas), ya que incluyó iniciativas culturales (por ejemplo, eventos artísticos que fomentan la participación de PCD). El segundo tema más mencionado es el de políticas públicas que los beneficien $(30,5 \%$ de presencia), el cual incluyó asuntos sobre programas sociales de ayuda a grupos vulnerables, tales como Bienevales, el programa de subsidio de transporte público del gobierno estatal.

Aunque el 73,6 \% de las notas abordan la discapacidad en general, también se observa que las discapacidades más mencionadas en las notas analizadas son, en orden de importancia, la visual, la de movilidad, la intelectual, la auditiva y la del habla. Aunque la discapacidad motriz es la más común en la población mexicana, esta no es la más mencionada ${ }^{3}$

Los actores más mencionados en las notas fueron los funcionarios públicos (54,4 \% de notas), seguidos por la categoría "otros", en la que se incluyen aquellos sujetos que no pudieron ser clasificados en las categorías precedentes: artistas, maestros, ingenieros, otros profesionistas y hasta ciudadanos comunes. El tercer tipo de actor más nombrado fue el de las ONG, académicos y empresarios (25,7 \% de notas), que sobrepasan a las PCD (24,4 \%).

Los problemas que aquejan a las PCD se atribuyen principalmente al gobierno y sus funcionarios, al incumplir las leyes o no implementar políticas públicas que beneficien a las PCD ( $22 \%$ de las notas analizadas); en segundo lugar, a la sociedad en general (12,5 \% de notas). El tercer lugar se reparte más o menos equitativamente entre las empresas y sus empleadores, por no generar oportunidades de empleo, y las instituciones educativas, por no gestionar

3 En Jalisco 226.142 habitantes tienen discapacidad motriz (Inegi, 2013). 
una educación inclusiva. El siguiente fragmento de la noticia titulada "Viven calvario” representa un ejemplo de atribución del problema al gobierno:

Hasta 10 horas, en medio de un desorden, demoraron adultos mayores y personas con discapacidad en el proceso de credencialización para recibir Bienevales. En el módulo ubicado en la Secretaría de Desarrollo e Integración Social (Sedis) —único hasta ayer en el que se podía realizar el proceso—, cientos de personas debían hacer fila para realizar el trámite. Todos pedían informes (Mural, 2015, 9 de enero).

Debe señalarse que las asociaciones u organizaciones de ayuda a PCD solo fueron señaladas como responsables del problema en cuatro notas $(0,7 \%)$. Un ejemplo es la noticia “Defienden 'jaulas' para discapacitados”, que aborda el informe de la organización Disability Rights International en el que se denuncian abusos cometidos en una casa hogar.

La principal solución que las notas analizadas sugieren o mencionan para los problemas de las PCD es la intervención del gobierno (46,8 \%), las oportunidades educativas (11,8\% de notas) y los avances médicos y tecnológicos (10,8 \%). Una solución son las acciones de los familiares y personas cercanas a la discapacidad (8,4 \%), la cual aparece en notas que buscan prevenir la discapacidad; es decir, abordan temas de prevención de accidentes o autocuidados en el embarazo.

La evaluación moral que se hace de la discapacidad es primordial-mente neutra (94\%), puesto que cantidades marginales de notas contienen evaluaciones positivas o negativas en torno a la discapacidad. Las evaluaciones morales en las notas sobre discapacidad podrían llegar a provocar emociones como compasión o reconocimiento en la audiencia.

\section{Discusión}

Los resultados de esta investigación permiten identificar las principales características de la representación mediática de las PCD en periódicos mexicanos, entre las que destacan, en primer lugar, (1) un paternalismo hacia las PCD: en el discurso periodístico predomina la idea de que el gobierno es totalmente responsable de los problemas que aquejan a las PCD y, en con- secuencia, de que deben ser los encargados de resolver sus problemas; esto, muchas veces de una forma asistencialista en vez de conforme a derechos y obligaciones. Esto no solo sucede con el gobierno, sino con la sociedad en general, a la que se atribuyen responsabilidades que, más allá de la solidaridad, implican piedad, misericordia y asistencia hacia las PCD. 
Este paternalismo ("tendencia a aplicar las formas de autoridad y protección propias del padre en la familia tradicional a relaciones sociales de otro tipo; políticas, laborales, etc.”, de acuerdo con el Diccionario de la Lengua Española de la RAE) se refleja en las notas que transmiten la idea de que el gobierno y la sociedad en general deben dar respuesta a los problemas de las PCD (independientemente de si es su obligación), lo cual a su vez revela una concepción de la PCD como persona desempoderada.

Este rasgo coincide con lo reportado por Haller y Burns (2015), quienes concluyeron en su análisis de la cobertura mediática de las PCD en Australia que la mayoría de las notas periodísticas siguen el modelo tradicional de patología social, el cual presenta a las PCD como sujetos en desventaja necesitados de un apoyo económico de parte del Estado o la sociedad que se concibe como caridad en vez de como un derecho (Haller \& Burns, 2015).

Una segunda característica (2) es la representación de las PCD como carentes de capacidad para resolver sus problemas. Los medios no representan a las PCD ni como generadoras de sus problemas ni como creadoras de soluciones para estos. Esto indica que los medios de comunicación conciben a las PCD como sujetos pasivos frente a estas situaciones, lo cual explica la gran responsabilidad que se adjudica al gobierno en la solución y creación de situaciones problemáticas para las PCD. El Teletón no parece contribuir de manera significativa a esta representación mediática, ya que esta fundación de ayuda para las PCD tuvo una frecuencia reducida de menciones.

El desfase existente entre el eslogan de lucha nada sobre nosotros sin nosotros y el lugar marginal que se da a la voz de las PCD en los contenidos periodísticos hace aún más vívida la imagen que se refleja de la PCD como un sujeto desempoderado incapaz incluso de hablar públicamente de sus propios problemas y sus experiencias. Una de las causas de lo anterior puede ser la concepción de las PCD como individuos fuera de la norma (Goffman, 2008), lo cual provoca la negación de sus derechos de expresión, pues al no ser “normales” no gozan de los mismos derechos.

En tercer lugar (3), los medios destacan formas e instituciones de socialización alternativas, pero poco incluyentes. Aunque no se incluyeron categorías en las que se abordarán los temas del deporte y el arte como parte de las actividades desarrolladas por las PCD que fungen como espacios de socialización recreativos y rehabilitadores, estos se codificaron dentro de la categoría “otros”, lo cual además explica la alta frecuencia de dicha categoría.

Este hallazgo demuestra que los procesos socializadores (Berger \& Luckmann, 1968) de las PCD enfatizados por las organizaciones de medios no son los regulares, como la escuela o el trabajo, sino alternativos, como el deporte 
adaptado, los centros educativos especiales —donde en la mayoría de los casos no se busca enseñar conocimiento académico sino oficios y actividades manuales-. Estos fungen como centros socializadores básicos en los que las PCD tendrían una interacción mayor con personas en su misma condición, lo cual no abona a su inclusión en la sociedad, sino a su integración en grupos con características definidas según condiciones particulares de discapacidad. Aunque tanto Berger y Luckmann como Goffman señalan que los espacios alternativos ayudan a las PCD a construir su identidad, esta se crea de forma deficiente, con antidefiniciones (Berger \& Luckmann, 1968) y alienaciones endogrupales que constituyen "la categoría que puede servir para su descrédito” (Goffman, 2008, p. 143). Esto no quiere decir que dichas formas de socialización sean malas, sino que son segregadoras y no permiten que las PCD se inserten en una vida social en la que coexistan con personas sin discapacidad.

Dicha representación de las PCD como necesitadas de espacios socializadores alternativos o rehabilitadores contribuye a mantener una concepción de la discapacidad acorde con el modelo médico: en este, los medios de comunicación son un "coro" (Berger \& Luckmann, 1968) que repite y reafirma el perfil de las PCD como sujetos con padecimientos que les impiden realizar las mismas actividades que las personas sin discapacidad. Los periódicos difunden primordialmente una representación mediática de las PCD como individuos con espacios de existencia distintos a los convencionales, pero dichos espacios alternativos no les permitirán desarrollar el resto de sus capacidades para lograr insertarse en la vida social (económica, educativa, afectiva, familiar) de forma plena. Como la rutina de normalización de la que habla Goffman (2008) no figura en las representaciones mediáticas, puede concluirse que una familiarización con las PCD parece aún lejana.

Una cuarta idea recurrente identificada dentro de la representación mediática de la discapacidad fue (4) la necesidad de oportunidades de empleo y educación para las PCD. Ambas áreas aparecen en los periódicos como las soluciones primordiales para los problemas de las PCD.

El propósito de esta investigación fue describir la representación mediática de las PCD para generar una reflexión sobre las formas en que esta condiciona su participación social. De acuerdo con este análisis, los medios representan a las PCD como sujetos absueltos (pues no se les responsabiliza de sus problemas ni de sus actos, ni se les involucra en las soluciones); intocables (dado que no se les critica, pero tampoco se les incluye, pues aparecen representados realizando actividades segregadas del resto de la población) y oprimidos (es decir, sin suficiente capacidad para resolver sus propios problemas, con lo que sus atributos como ciudadanos activos y acreedores de derechos y obligaciones se anula). 
Aunque este es un problema multifactorial, la representación mediática influye decisivamente, pues los contenidos de los medios enteran a las audiencias acerca del rango legítimo de problemas, soluciones y responsables de los problemas relacionados con la discapacidad, eximiendo a las PCD de responsabilidad sobre estos y, en consecuencia, quitándoles poder de acción sobre las soluciones posibles para dichos problemas.

Entre las limitaciones de esta investigación debe señalarse el hecho de que solo se analizaron periódicos en sus versiones impresas y digitales y se dejó por fuera la televisión, la radio y los contenidos de ficción. Aunque la muestra de periódicos no representa estadísticamente la cobertura periodística en México, el muestreo de relevancia comprende a los periódicos de prestigio de la segunda ciudad más poblada del país.

La investigación sobre la representación mediática de la discapacidad debe continuar, ya que la discapacidad cada vez se vuelve más visible en el mundo, en parte por los avances médicos y sociales que permiten el diagnóstico oportuno y el registro con cada vez mayor precisión de las condiciones de discapacidad. Ante tal panorama, el estudio social de la discapacidad no debe atrasarse, ya que sirve como fundamento de los modelos incluyentes en el mundo verdadero, fuera de los hospitales, de las instituciones asistencia- listas y de las escuelas especiales, los cuales no son necesariamente modelos de inclusión malos sino más bien insuficientes para que una persona crezca, se desarrolle y florezca plenamente en un mundo que la acepte y valore sus aportaciones. Los hallazgos de esta investigación abonan a un mejor entendimiento de la discapacidad no solo como una condición médica sino como una condición social. Los medios de comunicación son instrumentales para construir y diseminar las diversas concepciones que hay de la discapacidad, al ser una de las principales fuentes de información de las que el público se sirve para construir sus propias concepciones sobre el mundo y sobre lo que en él hay.

Una forma en que las PCD pueden alcanzar visibilidad y ser reconocidas como un sector de la población que importa, tiene necesidades y capacidades para aportar al bienestar social y común es representarlos en los medios como ciudadanos con derechos y obligaciones, que tienen entre sus actividades el trabajo, el estudio y el entretenimiento, y no solo como un grupo de seres extraordinarios cuyas únicas oportunidades de desarrollo son las áreas deportivas y artísticas. Sin embargo, esto por sí solo no bastará para empoderar a las PCD, ni exonera al Estado de cumplir con sus obligaciones de garantizar su inclusión y su acceso a la salud, educación e información. 


\section{References}

Berger, P., \& Luckmann, T. (1968). La construcción social de la realidad, Buenos Aires: Amorrortu.

Carter, N., \& Williams, J. (2012). 'A genuinely emotional week’: learning disability, sport and television-notes on the Special Olympics GB National Summer Games 2009. Media, Culture \& Society 34(2), 211-227.

Comité sobre los Derechos de las Personas con Discapacidad (2014). Observaciones finales sobre el informe inicial de México. CRPD/C/MEX/ CO/1.

Cuesta et al. (2015). Percepciones sobre la discapacidad transmitidas por la prensa escrita española durante 1978, 1995 y 2007 [Perceptions about Disability Transmitted by Spanish Written Press during 1978, 1995 and 2007]. Estudios sobre el Mensaje Periodístico 21(1), 269.

Ellis, K. (2008). Beyond the Aww Factor: Human interest Profiles of Paralympians and the media navigation of physical difference and social stigma. Asia Pacific Media Educator, (19), 23.

Entman, R. (1993). Framing: Toward clarification of a fractured paradigm. Journal of Communication 43(4), 51-58.

Espinola-Nadurille, M. \& Delgado, G. (2009). Mental disability and discriminatory practices: effects of social representations of the Mexican population. International Journal of Social Psychiatry 55(3), 238-246.

Goffman, E. (2008). Estigma. La identidad deteriorada. Buenos Aires: Amorrortu.

Haller, B., Dorries, B., \& Rahn, J. (2006). Media labeling versus the US disability community identity: a study of shifting cultural language. Disability \& Society 21(1), 61-75.

Haller, B., \& Burns, S. (2015). The Politics of Representing Disability. Exploring News Coverage of the Americans with Disabilities Act and the National Disability Insurance Scheme. Asia Pacific Media Educator 25(2) 262-277. University of Wollongong, Australia.

Holton, A. (2013). What's Wrong With Max? Parenthood and the Portrayal of Autism Spectrum Disorders. Journal of Communication Inquiry 37(1), 45-63.

Howe, P. (2008). From inside the newsroom: Paralympic media and the production of elite disability. International Review for the Sociology of Sport 43(2), 135-150.

Huérfano, V. (2015, 9 de enero). Viven calvario. Mural. Recuperado de: [https:// www.mural.com/] 
Iáñez-Domínguez, A., y González, B. (2017). The Representation of Physical Functional Diversity in the Spanish Press. Communication \& Society 30(1), 105-124.

Instituto Nacional de Estadística y Geografía (Inegi) (2013). Las personas con discapacidad en México, una visión al 2010. México.

Iyengar, S. (1990). Framing responsibility for political issues: The case of poverty. Political Behavior 12(1), 19-40.

Maia, R. \& Vimieiro, A. (2015). Recognition and moral progress: a case study about discourses on disability in the media. Political Studies, 63(1), 161- 180 .

McKeever, B. (2013). News Framing of Autism: Understanding Media Advocacy and the Combating Autism Act. Science Communication, 35.

Misener, L. (2013). A media frames analysis of the legacy discourse for the 2010 Winter Paralympic Games. Communication \& Sport, 1(4), 342-364.

Rimmerman, A., \& Herr S. (2004). The Power of the Powerless: A Study on the Israeli Disability Strike of 1999. Journal of Disability Policy Studies 15,12. Publicado por Hammill Institute on Disabilities y Sage Publications.

Rodelo, F., y Muñiz, C. (2016). Uso del análisis de conglomerados para la detección de encuadres en el tratamiento periodístico de la Iniciativa Mérida. Comunicación y Sociedad, 27, 53-77.

Shakespeare, T. (2006). The Social Model of Disability. Davis, L. J. The Disability Studies Reader. Routledge.

Silva, C., \& Howe, P. (2012). The (in) validity of supercrip representation of Paralympian athletes. Journal of Sport and Social Issues 36(2), 174-194.

Von Krogh, T. (2010). From a medical to a human rights perspective. A case study of efforts to change the portrayal of persons with disabilities on Swedish television. International Communication Gazette 72(4-5), 379- 394.

Wimmer, R. \& Dominick, J. (2001). Introducción a la investigación de medios masivos de comunicación. México: Thomson.

Zhang, L. \& Haller, B. (2013). Consuming image: How mass media impact the identity of people with disabilities. Communication Quarterly, 61(3), 319-334. 